\title{
ESTIMATES FOR THE MAXIMAL OPERATOR OF THE ORNSTEIN-UHLENBECK SEMIGROUP
}

\author{
CRISTIAN E. GUTIÉRREZ AND WILFREDO O. URBINA
}

(Communicated by J. Marshall Ash)

\begin{abstract}
We show pointwise estimates for the maximal operator of the Ornstein-Uhlenbeck semigroup for functions that are integrable with respect to the Gaussian measure. The estimates are used to prove pointwise convergence.
\end{abstract}

The Ornstein-Uhlenbeck semigroup is defined by

$$
T_{t} f(x)=\int_{R^{n}} k(t, x, y) f(y) d y,
$$

where

$$
k(t, x, y)=\pi^{-n / 2}\left(1-e^{-2 t}\right)^{-n / 2} \exp \left(-\frac{\left|e^{-t} x-y\right|^{2}}{1-e^{-2 t}}\right), \quad t>0, x \in R^{n} .
$$

This family of operators was considered by L. S. Ornstein and G. E. Uhlenbeck to construct a theory of Brownian motion [N]. The infinitesimal generator of this semigroup is $L=\frac{1}{2} \Delta-x \cdot \nabla$, and the eigenfunctions are the Hermite polynomials. If $0<r<1$ then the Poisson-Hermite integral of the function $f$ is given by

$$
P_{r} f(x)=T_{\log \frac{1}{r}} f(x) .
$$

For $x \in R^{n}$ we set $\gamma(x)=\pi^{-n / 2} e^{-|x|^{2}}$, and given $p \geq 1$, by $L^{p}\left(R^{n}, \gamma\right)$ we denote the class of functions that are integrable to the $p$ th power over $R^{n}$ with respect to the measure $\gamma(x) d x$. Also, $\|\cdot\|_{p, \gamma}$ denotes the usual norm. Let

$$
P^{*} f(x)=\sup _{0<r<1}\left|P_{r} f(x)\right| .
$$

In one dimension the maximal operator $P^{*}$ was studied by B. Muckenhoupt [M] in 1969 and, independently, by C. P. Calderón [C] also in 1969. They showed that $P^{*}$ is bounded in $L^{p}(R, \gamma)$ if $p>1$ and is of weak-type $(1,1)$ with respect to $\gamma$. C. P. Calderón [C], by adapting the well-known results of Abel and Cesàro summability of the multiple Fourier series to the case of Abel

Received by the editors March 5, 1990 and, in revised form, July 12, 1990.

1991 Mathematics Subject Classification. Primary 42B25; Secondary 47D06, $60 \mathrm{~J} 60$.

The first author was supported in part by NSF Grant \#DMS-9003095. 
summability of multiple Hermite series, (see [Z]) has shown the boundedness in $L^{p}\left(R^{n}, \gamma\right), p>1$, of $P^{*}$ with respect to the Gaussian measure.

This paper concerns the behavior of $P^{*}$ in $L^{1}\left(R^{n}, \gamma\right)$. We introduce the centered maximal function defined by

$$
M_{\gamma} f(x)=\sup _{r>0} \frac{1}{\gamma\left(B_{r}(x)\right)} \int_{B_{r}(x)}|f(y)| \gamma(y) d y,
$$

$B_{r}(x)$ is the ball in $R^{n}$ with center $x$ and radius $r$, and $\gamma\left(B_{r}(x)\right)=\int_{B_{r}(x)} \gamma(t) d t$. The standard proof using the Besicovitch covering lemma shows that $M_{\gamma}$ is of weak-type $(1,1)$ with respect to the measure $\gamma d x$.

In this paper we show the following:

Theorem. If $f \in L^{1}\left(R^{n}, \gamma\right)$ then the following pointwise estimate holds:

$$
P^{*} f(x) \leq c_{n} M_{\gamma} f(x)+(2 \vee|x|)^{n} e^{|x|^{2}}\|f\|_{1, \gamma} .
$$

Also $P_{r} f \rightarrow f$ as $r \rightarrow 1^{-}$in $L^{1}\left(R^{n}, \gamma\right)$ and for almost every $x$ we have

$$
P_{\sqrt{1-s}} f(y / \sqrt{1-s}) \rightarrow f(x),
$$

as $s \rightarrow 0$ for $|x-y| \leq s^{1 / 2}$.

In one dimension our theorem gives a new proof of the weak-type $(1,1)$ of $P^{*}$ proved in $[\mathrm{C}, \mathrm{M}]$. This follows because the function $(2 \vee|x|) e^{|x|^{2}}$ belongs to weak $L^{1}(R, \gamma)$; see (5) below.

Proof of the theorem. We may assume $f \geq 0$. If we set

$$
u(y, s)=s^{-n / 2} \int_{R^{n}} \gamma\left(\frac{y-z}{s^{1 / 2}}\right) f(z) d z,
$$

then

$$
P^{*} f(x)=\sup _{0<t<1} u(\sqrt{1-t} x, t)
$$

Let

$$
P_{1}^{*} f(x)=\sup _{0<t<1 /|x|^{2} \wedge 1 / 4} u(\sqrt{1-t} x, t)
$$

and

$$
P_{2}^{*} f(x)=\sup _{1 /|x|^{2} \wedge 1 / 4 \leq t<1} u(\sqrt{1-t} x, t) .
$$

We introduce the maximal function

$$
M f(x)=\sup _{(y, s) \in \Gamma(x)} u(y, s),
$$

where $\Gamma(x)=\left\{(x, s):|x-y| \leq s^{1 / 2}, 0<s<1 /|x|^{2} \wedge 1 / 4\right\}$.

We shall show that

$$
P_{1}^{*} f(x) \leq M f(x) \leq c_{n} M_{\gamma} f(x)
$$


and

$$
P_{2}^{*} f(x) \leq(2 \vee|x|)^{n} e^{|x|^{2}}\|f\|_{1, \gamma} .
$$

The first inequality in (1) follows because if $0<t<1 /|x|^{2} \wedge 1 / 4$ then $(\sqrt{1-t} x, t) \in \Gamma(x)$.

Now set $a_{j}=j^{1 / 2}$ for $j=1,2, \ldots$. We write

$$
u(y, s)=s^{-n / 2} \sum_{j=1}^{\infty} \int_{a_{j-1} s^{1 / 2} \leq|y-z|<a_{j} s^{1 / 2}} \gamma\left(\frac{y-z}{s^{1 / 2}}\right) f(z) d z .
$$

If $(y, s) \in \Gamma(x)$ and $|y-z|<a_{j} s^{1 / 2}$ then $|x-z|<\left(1+a_{j}\right) s^{1 / 2}$ and consequently,

$$
u(y, s) \leq s^{-n / 2} \sum_{j=1}^{\infty} \gamma\left(a_{j-1}\right) \int_{|x-z|<\left(1+a_{j}\right) s^{1 / 2}} f(z) d z .
$$

We have $|z|^{2}=|z-x|^{2}+2 x \cdot(z-x)+|x|^{2}$, hence

$$
\begin{aligned}
& \int_{|x-z|<\left(1+a_{j}\right) s^{1 / 2}} f(z) d z \\
& \leq \exp \left(|x|^{2}+2|x|\left(1+a_{j}\right) s^{1 / 2}+\left(1+a_{j}\right)^{2} s\right) \int_{|x-z|<\left(1+a_{j}\right) s^{1 / 2}} f(z) e^{-|z|^{2}} d z \\
& \leq \exp \left(|x|^{2}+2|x|\left(1+a_{j}\right) s^{1 / 2}+\left(1+a_{j}\right)^{2} s\right) M_{\gamma} f(x) \int_{|x-z|<\left(1+a_{j}\right) s^{1 / 2}} e^{-|z|^{2}} d z \\
&= \exp \left(2|x|\left(1+a_{j}\right) s^{1 / 2}+\left(1+a_{j}\right)^{2} s\right) M_{\gamma} f(x) \\
& \times \int_{|x-z|<\left(1+a_{j}\right) s^{1 / 2}} e^{-|z-x|^{2}-2 x \cdot(z-x)} d z \\
& \leq \exp \left(4|x|\left(1+a_{j}\right) s^{1 / 2}+\left(1+a_{j}\right)^{2} s\right) M_{\gamma} f(x) \int_{|z|<\left(1+a_{j}\right) s^{1 / 2}} e^{-|z|^{2}} d z \\
& \leq \Omega_{n}\left(1+a_{j}\right)^{n} s^{n / 2} \exp \left(4|x|\left(1+a_{j}\right) s^{1 / 2}+\left(1+a_{j}\right)^{2} s\right) M_{\gamma} f(x) .
\end{aligned}
$$

By adding in $j$ we get for $(y, s) \in \Gamma(x)$

$$
u(y, s) \leq c_{n} M_{\gamma} f(x),
$$

and then the second inequality in (1) follows.

To prove (2) observe that if $0<t<s$ and $x, y \in R^{n}$ then for any $z \in R^{n}$ we have

$$
\frac{|y-z|^{2}}{s}-\frac{|x-z|^{2}}{t} \leq \frac{|y-x|^{2}}{s-t} \text {. }
$$

Thus

$$
u(y, t) \leq u(x, s)\left(\frac{s}{t}\right)^{n / 2} \exp \left(\frac{|x-y|^{2}}{s-t}\right),
$$

for $0<t<s$. In particular

$$
u(\sqrt{1-t} x, t) \leq u(0,1)(1 / t)^{n / 2} e^{|x|^{2}}
$$


for $0<t<1$. If $t \geq 1 /|x|^{2} \wedge 1 / 4$ then $1 / t \leq(2 \vee|x|)^{2}$ and hence (2) follows.

The convergence in $L^{1}$ is proved in the same way as in the proof of Theorem 2 of $[\mathrm{M}]$.

To show the a.e. convergence define

$$
\Omega f(x)=\lim _{\alpha \rightarrow 0}\left(\sup _{(y, s) \in \Gamma(x), 0<s<\alpha}|u(y, s)-f(x)|\right),
$$

and set

$$
f=f \phi_{k}+f\left(1-\phi_{k}\right)=f_{1}+f_{2},
$$

where $k$ is an integer greater than 1 , and $\phi_{k}$ is the characteristic function of the ball $B_{k}(0)$. We shall show that

$$
\Omega f(x) \leq c_{n} M_{\gamma} f_{2}(x)
$$

for every $k>1$ and almost every $x$ with $|x| \leq k-1$.

We have that

$$
\lim _{r \rightarrow 0} \frac{1}{\gamma\left(B_{r}(x)\right)} \int_{B_{r}(x)}|f(z)-f(x)| e^{-|z|^{2}} d z=0,
$$

for almost every $x$. Let $x$ be a point satisfying (4), then for every $\varepsilon>0$ there exists $\delta, 0<\delta<1$ such that

$$
\frac{1}{\gamma\left(B_{r}(x)\right)} \int_{B_{r}(x)}|f(z)-f(x)| e^{-|z|^{2}} d z<\varepsilon
$$

for $0<r<\delta$. Set

$$
g(z)= \begin{cases}f(z)-f(x), & \text { if }|x-z| \leq \delta, \\ 0, & \text { if }|x-z|>\delta,\end{cases}
$$

and notice that $M_{y} g(x)<\varepsilon$. If we set $u_{i}(y, s)=\gamma_{s^{1 / 2}} * f_{i}(y), i=1,2$ then we have

We write

$$
u(y, s)-f(x)=u_{1}(y, s)-f_{1}(x)+u_{2}(y, s)-f_{2}(x) .
$$

$$
\begin{aligned}
u_{1}(y, s)-f_{1}(x) & =\pi^{-n / 2} s^{-n / 2} \int_{R^{n}} \gamma\left(\frac{y-z}{s^{1 / 2}}\right)\left(f_{1}(z)-f_{1}(x)\right) d z \\
& =\pi^{-n / 2} s^{-n / 2}\left(\int_{|x-z| \leq \delta}+\int_{|x-z|>\delta}\right) \\
& =I+I I .
\end{aligned}
$$

If $|x| \leq k-1$ and $(y, s) \in \Gamma(x)$ then by (1) we have

$$
|I| \leq M g(x) \leq c_{n} M_{\gamma} g(x)<c_{n} \varepsilon .
$$

To estimate $I I$, observe that if $|x-y| \leq s^{1 / 2}$ and $\delta \geq 2 s^{1 / 2}$ then

$$
\begin{aligned}
|I I| \leq & \pi^{-n / 2} s^{-n / 2} \int_{|y-z| \geq \delta / 2} e^{-|y-z|^{2} / s}\left|f_{1}(z)\right| d z \\
& +\pi^{-n / 2} s^{-n / 2} \int_{|x-z|>\delta} e^{-|y-z|^{2} / s}\left|f_{1}(x)\right| d z \\
= & I I_{1}+I I_{2} .
\end{aligned}
$$


We have

$$
\begin{aligned}
I I_{1} & =\pi^{-n / 2} s^{-n / 2} \int_{\{|y-z| \geq \delta / 2,|z| \leq k\}} \exp \left(-\frac{|y-z|^{2}}{s}+|z|^{2}\right)|f(z)| e^{-|z|^{2}} d z \\
& \leq \pi^{-n / 2} s^{-n / 2} \exp \left(\left(-\delta^{2} / 4 s\right)+k^{2}\right)\|f\|_{1, \gamma}
\end{aligned}
$$

and

$$
\begin{aligned}
I I_{2} & =\left|f_{1}(x)\right| \pi^{-n / 2} s^{-n / 2} \int_{|x-y+t| \geq \delta} e^{-|t|^{2} / s} d t \\
& \leq\left|f_{1}(x)\right| \pi^{-n / 2} s^{-n / 2} \int_{|t| \geq \delta / 2} e^{-|t|^{2} / s} d t \\
& =\left|f_{1}(x)\right| \pi^{-n / 2} \int_{|x| \geq \delta / 2 s^{1 / 2}} e^{-|x|^{2}} d x .
\end{aligned}
$$

If $|x| \leq k$ then $f_{2}(x)=0$ and consequently by (1)

$$
\left|u_{2}(y, s)-f_{2}(x)\right| \leq c_{n} M_{\gamma} f_{2}(x)
$$

for $(y, s) \in \Gamma(x)$.

Therefore, collecting estimates and taking supremum over $(y, s) \in \Gamma(x)$, $0<s<\alpha$, and letting $\alpha \rightarrow 0$ we obtain

$$
\Omega f(x) \leq c_{n}\left(\varepsilon+M_{\gamma} f_{2}(x)\right),
$$

for every $\varepsilon>0$, and a.e. $x$ with $|x| \leq k-1$, which gives (3). Given $\varepsilon>0$, we pick $k$ such that $\left\|f_{2}\right\|_{1, \gamma}<c \varepsilon^{2}$ then by $(3)$ and the weak-type $(1,1)$ of $M_{\gamma}$ we obtain

$$
\gamma\{x:|x| \leq k-1, \Omega f(x)>\varepsilon\}<\varepsilon,
$$

which implies $\Omega f(x)=0$ almost everywhere.

Remarks. (A) Set $\varphi(x)=(2 \vee|x|)^{n} e^{|x|^{2}}$ and $E_{\lambda}=\{x: \varphi(x)>\lambda\}, \lambda>0$. We shall show that

$$
\gamma\left(E_{\lambda}\right) \leq c\left(1+\ln ^{+} \lambda\right)^{n-1} / \lambda
$$

Since $\gamma$ is a finite measure, it is enough to prove (5) for $\lambda$ large. The function $\varphi$ increases with $|x|$ then $E_{\lambda}=\left\{x:|x| \geq r_{0}\right\}$ where $r_{0}^{n} \exp \left(r_{0}^{2}\right)=\lambda$. Therefore $r_{0} \sim(\ln \lambda)^{1 / 2}$ for $\lambda$ large. Hence

$$
\begin{aligned}
\gamma\left(E_{\lambda}\right) & =c_{n} \int_{r_{0}}^{\infty} r^{n-1} e^{-r^{2}} d r \\
& =c_{n} e^{-r_{0}^{2}} \int_{0}^{\infty}\left(t+r_{0}^{2}\right)^{(n / 2)-1} e^{-t} d t \\
& =c_{n} e^{-r_{0}^{2}} r_{0}^{n-2} \int_{0}^{\infty}\left(1+t / r_{0}^{2}\right)^{(n / 2)-1} e^{-t} d t \\
& \sim c_{n} e^{-r_{0}^{2}} r_{0}^{n-2}=c_{n} r_{0}^{2(n-1)} / \lambda,
\end{aligned}
$$

and (5) follows. 
(B) The theorem implies that $P_{r}(x) \rightarrow f(x)$ as $r \rightarrow 1^{-}$a.e. This last result can also be obtained as a consequence of Lemma (2.17) of [C]. The onedimensional case is due to $\mathrm{E}$. Hille $[\mathrm{H}]$.

\section{REFERENCES}

[C] C. P. Calderón, Some remarks on the multiple Weierstrass transform and Abel summability of multiple Fourier-Hermite series, Studia Math. 32 (1969), 119-148.

[H] E. Hille, A class of reciprocal functions, Ann. of Math. (2) 27 (1926), 426-464.

[M] B. Muckenhoupt, Poisson integrals for Hermite and Laguerre expansions, Trans. Amer. Math. Soc. 139 (1969), 231-242.

[N] E. Nelson, Dynamical theories of Brownian motion, Princeton Univ. Press, Princeton, NJ, 1967.

[Z] A. Zygmund, Trigonometric series, Cambridge Univ. Press, 1959.

Department of Mathematics, Temple University, Philadelphia, Pennsylvania 19122

Departamento de Matemática, Universidad Central de Venezuela, Caracas, VeNEZUELA 